\title{
A new Fibonacci type collocation procedure for boundary value problems
}

\author{
Ayşe Betül Koç ${ }^{1 *}$, Musa Çakmak², Aydın Kurnaz ${ }^{1}$ and Kemal Uslu ${ }^{1}$
}

\author{
"Correspondence: \\ aysebetulkoc@selcuk.edu.tr \\ 1 Department of Mathematics, \\ Faculty of Science, Selcuk University, \\ Konya, Turkey \\ Full list of author information is \\ available at the end of the article
}

\begin{abstract}
In this study, we present a new procedure for the numerical solution of boundary value problems. This approach is mainly founded on the Fibonacci polynomial expansions, the so-called pseudospectral methods with the collocation method. The applicability and effectiveness of our proposed approach is shown by some illustrative examples. Then, the results indicate that this method is very effective and highly promising for linear differential equations defined on any subinterval of the real domain.
\end{abstract}

MSC: $35 \mathrm{~A} 25$

Keywords: ordinary differential equations; spectral methods; collocation method; Fibonacci polynomials

\section{Introduction}

A lot of applications, which are of fundamental interest in numerical analysis, have been made on the expansion of an analytic function $f(x)$ in a series of the form

$$
f(x)=\sum_{k=0}^{\infty} a_{k} \varphi_{k}(x)
$$

where the set $\left\{\varphi_{k}(x)\right\}$ is a special trial polynomial (or function) basis and $a_{k}$ 's are constant coefficients [1-9]. One of the main utilization areas of the expansions with different basis can be seen in the solution methods for the differential equations. The idea of finding the solution of a differential equation in form (1.1) goes back, at least, to Lanczos (1938) [10,11]. Then, three main techniques have been evolved from that idea, and each of these techniques has its own advantages in the implementation (details and applications of those can be seen in [11-13]). Here, we deal only with the pseudospectral (collocation) type method. In the pseudospectral method, the solution of a differential equation is expressed as a linear combination of the polynomials in the basis set. Therefore, the coefficients in the combination are determined by the use of certain discrete points called collocation points. Thus, the accuracy of the approximation and the efficiency of its implementation are closely related to the choice of the grid points and the basis set. Determination of an appropriate set of basis should be kept in view of some rules. The most common one of those rules is that the geometry or the field of applicability determines the basis set. Then, collocation points are chosen according to the basis (for details see [12]). For example, 
the well-known basis functions of the Fourier expansion $\{1, \cos (n x), \sin (n x), \ldots\}$ are all periodic. Thus, Fourier expansion is good for the solutions of the problems with periodic behaviors [12-14]. On the other hand, since the Chebyshev polynomials of the first kind and Legendre polynomials are orthogonal in the interval $[-1,1]$, non-periodic problems on the range of $[-1,1]$ can be solved by the collocation method, the matrix method or the Tau method [15-23]. However, when the problem is posed on an unbounded interval, alternative strategies are developed for the solution, such as domain truncation [24] and choosing a basis functions intrinsic to an infinite interval as Sinc [25], Hermite [26], and exponential Chebyshev [27]) or to semi-infinite interval as rational Chebyshev [28-30], and Laguerre [31].

In this work, our aim is to develop a new type of collocation method for the boundary value problems (BVPs) on any subinterval of the real axis requiring no domain translation. For this reason, Fibonacci polynomials that have, so far, never been used as a basis for the collocation are considered. Even though this new approach is mainly intended for the BVPs, it can be successfully implemented on the initial value problems (IVPs).

Byrd [32] has defined the set $\left\{\varphi_{k}(t)\right\}(k=0,1, \ldots)$ as Fibonacci polynomials that satisfy the recurrence relation

$$
\varphi_{k+2}(t)-2 t \varphi_{k+1}(t)-\varphi_{k}(t)=0, \quad-\infty<t<\infty
$$

with the initial conditions

$$
\varphi_{0}(t)=0, \quad \varphi_{1}(t)=1 .
$$

He has also noted that in the special case of $t=\frac{1}{2}$, the relation above with the initial conditions reduces to the recurrence equation of Fibonacci numbers denoted by $\varphi_{k}\left(\frac{1}{2}\right)$ or $F_{k}$. However, equation (1.2) with conditions (1.3) has a wide use as the relation of Pell polynomials [33]. Therefore, the well-known and common recurrence relation for the Fibonacci polynomials $\left\{F_{k}(x)\right\}(k=0,1, \ldots)$ is given [34]

$$
F_{k+2}(x)-x F_{k+1}(x)-F_{k}(x)=0
$$

with the initial conditions $F_{0}(x)=0, F_{1}(x)=1$. It is also noteworthy that the polynomial $\varphi_{k}(t)$ turns into $F_{k}(x)$ when the variable change of $t=\frac{x}{2}$ is used in equation (1.2).

Byrd [32] has investigated some fundamental properties and certain applications for the expansion of an analytic function in a series of basic set of Fibonacci polynomials. Moreover, many other important properties of those polynomials are also studied by Falcon and Plaza in $[34,35]$ and references therein.

\section{Properties of the Fibonacci polynomials}

The $k$-Fibonacci numbers and polynomials have been defined as follows:

Definition 1 [34] For any positive real number $k$, the $k$-Fibonacci sequence is defined recurrently by

$$
F_{k, n+1}=k F_{k, n}+F_{k, n-1}, \quad n \geq 1
$$


with the initial conditions

$$
F_{k, 0}=0 ; \quad F_{k, 1}=1 .
$$

Definition 2 [34] If $k$ is a real variable $x$ in equation (2.1), then it is obvious that $F_{k, n}=F_{x, n}$. Therefore, the corresponding Fibonacci polynomials are given by the following general formula

$$
F_{n+1}(x)= \begin{cases}1, & \text { if } n=0, \\ x, & \text { if } n=1, \\ x F_{n}(x)+F_{n-1}(x), & \text { if } n>1,\end{cases}
$$

from which the first few Fibonacci polynomials can be deduced as

$$
\begin{aligned}
& F_{1}(x)=1, \\
& F_{2}(x)=x, \\
& F_{3}(x)=x^{2}+1, \\
& F_{4}(x)=x^{3}+2 x,
\end{aligned}
$$

The next proposition indicates the relation between the derivatives of the Fibonacci polynomials followed by the integral equation.

Proposition 1 [35] The equality

$$
F_{n}(x)=\frac{1}{n}\left[F_{n+1}^{\prime}(x)+F_{n-1}^{\prime}(x)\right]
$$

holds for all natural numbers $n$. Thus, in view of (2.3), it is easy to verify the integral equation

$$
\int_{0}^{x} F_{n}(x) d x=\frac{1}{n}\left\{F_{n+1}(x)+F_{n-1}(x)-F_{n+1}(0)-F_{n-1}(0)\right\} .
$$

If $n$ is even, it can be seen that $F_{n+1}(0)=F_{n-1}(0)=1$ and, for odd n's, we write $F_{n+1}(0)=$ $F_{n-1}(0)=0$.

\section{Function approximation}

Now, suppose that $f(x)$ is a continuous function that can be expressed in terms of the Fibonacci polynomials

$$
f(x)=\sum_{r=1}^{\infty} a_{r} F_{r}(x)
$$

Then, a truncated expansion of $N$ Fibonacci polynomials can be written

$$
f(x) \cong \sum_{r=1}^{N} a_{r} F_{r}(x)=\mathbf{F}(x) \mathbf{A},
$$


where the Fibonacci row vector $\mathbf{F}(x)$ and the Fibonacci coefficient column vector $\mathbf{A}$ are given, respectively, by

$$
\begin{aligned}
& \mathbf{F}(x)=\left[\begin{array}{llll}
F_{1}(x) & F_{2}(x) & \cdots & F_{N}(x)
\end{array}\right], \\
& \mathbf{A}=\left[\begin{array}{llll}
a_{1} & a_{2} & \cdots & a_{N}
\end{array}\right]^{T} .
\end{aligned}
$$

\section{Matrix relations of the derivatives of approximation}

The $k$ th order derivative of the function (2.5) can be written as

$$
f^{(k)}(x)=\sum_{r=1}^{\infty} a_{r}^{(k)} F_{r}(x)
$$

When the infinite sum is truncated to $N$ terms, we get the approximation

$$
f^{(k)}(x) \cong \sum_{r=1}^{N} a_{r}^{(k)} F_{r}(x)=\mathbf{F}(x) \mathbf{A}^{(k)}, \quad k=0,1, \ldots, n,
$$

where $a_{r}^{(0)}=a_{r}, f^{(0)}(x)=f(x)$ and

$$
\mathbf{A}^{(k)}=\left[\begin{array}{llll}
a_{1}^{(k)} & a_{2}^{(k)} & \cdots & a_{N}^{(k)}
\end{array}\right]
$$

shows the coefficient vector of the polynomial approximation of $k$ th order derivative.

Proposition 2 Let $f(x)$ and kth order derivative be the functions given by (2.6) and (2.9), respectively. Then, there exists a relation between the Fibonacci coefficient matrices as

$$
\mathbf{A}^{(k+1)}=\mathbf{D}^{k} \mathbf{A}, \quad k=0,1, \ldots, n,
$$

where $\mathbf{D}$ is $N \times N$ operational matrix for the derivative defined by

$$
\mathbf{D}=\left[d_{i, j}\right]= \begin{cases}i \cdot \sin \frac{(j-i) \pi}{2}, & j>i, \\ 0, & j \leq i .\end{cases}
$$

Proof By using the integral relation (2.4), the Fibonacci coefficients $a_{r}^{(k)}$ and $a_{r}^{(k+1)}$ hold the equality,

$$
a_{r}^{(k)}=\frac{a_{r-1}^{(k+1)}}{r-1}+\frac{a_{r+1}^{(k+1)}}{r+1} .
$$

Therefore, it is easy to see the following recursive relations

$$
\begin{aligned}
& a_{r+1}^{(k)}=\frac{a_{r}^{(k+1)}}{r}+\frac{a_{r+2}^{(k+1)}}{r+2}, \\
& -a_{r+3}^{(k)}=-\frac{a_{r+2}^{(k+1)}}{r+2}-\frac{a_{r+4}^{(k+1)}}{r+4},
\end{aligned}
$$




$$
a_{r+5}^{(k)}=\frac{a_{r+4}^{(k+1)}}{r+4}+\frac{a_{r+6}^{(k+1)}}{r+6}
$$

Then, summing the terms on both sides gives

$$
\frac{a_{r}^{(k+1)}}{r}=a_{r+1}^{(k)}-a_{r+3}^{(k)}+a_{r+5}^{(k)}-a_{r+7}^{(k)}+\cdots
$$

or, equivalently,

$$
a_{r}^{(k+1)}=r \sum_{i=0}^{\infty}(-1)^{i} a_{r+2 i+1}^{(k)}, \quad r=1,2, \ldots, N
$$

Now, when we assume $a_{r}^{(k)}=0$ for $r>N$, the system (2.14) can be transformed into the matrix form,

$$
\mathbf{A}^{(k+1)}=\mathbf{D A}^{(k)}, \quad k=0,1, \ldots, n .
$$

Thus, using equality (2.15) allows us to write

$$
\begin{aligned}
& \mathbf{A}^{(1)}=\mathbf{D A}, \\
& \mathbf{A}^{(2)}=\mathbf{D A}^{(1)}=\mathbf{D}^{2} \mathbf{A}, \\
& \mathbf{A}^{(3)}=\mathbf{D A}^{(2)}=\mathbf{D}^{3} \mathbf{A}, \\
& \vdots \\
& \mathbf{A}^{(k)}=\mathbf{D}^{k} \mathbf{A},
\end{aligned}
$$

where $\mathbf{A}^{(0)}=\mathbf{A}$.

Corollary From equations (2.9) and (2.11), it is clear that the kth order derivative of the function can be expressed in terms of the Fibonacci coefficients as follows

$$
f^{(k)}(x)=\mathbf{F}(x) \mathbf{D}^{k} \mathbf{A}, \quad k=0,1, \ldots, n
$$

\section{Solution procedure for the ODE's}

Consider the general linear homogeneous differential equation of $n$th order,

$$
\sum_{k=0}^{n} q_{k}(x) y^{(k)}(x)=g(x)
$$

To apply our proposed procedure to the problem, we assumed that the unknown function $y(x)$ and its derivatives have similar forms as given in (2.16). Then, we define the collocation points so that

$$
x_{i}=a+\frac{b-a}{N-1}(i-1), \quad i=1,2, \ldots, N, a \leq x_{i} \leq b .
$$


Substituting these points (3.2) into the problem (3.1) gives

$$
\sum_{k=1}^{n} q_{k}\left(x_{i}\right) y^{(k)}\left(x_{i}\right)=g\left(x_{i}\right), \quad i=1,2, \ldots, N
$$

The system (3.3) can be, alternatively, written in the matrix form

$$
\sum_{k=1}^{n} \mathbf{Q}_{k} \mathbf{Y}^{(k)}=\mathbf{G}, \quad n \leq N
$$

where

$$
\mathbf{Q}_{k}=\left[\begin{array}{cccc}
Q_{k}\left(x_{1}\right) & 0 & \cdots & 0 \\
0 & Q_{k}\left(x_{2}\right) & \cdots & 0 \\
\vdots & \vdots & \ddots & \vdots \\
0 & 0 & \cdots & Q_{k}\left(x_{N}\right)
\end{array}\right] \text { and } \mathbf{G}=\left[\begin{array}{llll}
g\left(x_{1}\right) & g\left(x_{2}\right) & \cdots & g\left(x_{N}\right)
\end{array}\right]^{T} \text {. }
$$

Therefore, $k$ th order derivatives of the unknown function at the collocation points can be written in the matrix form as

$$
\left[y^{(k)}\left(x_{i}\right)\right]=\mathbf{F}\left(x_{i}\right) \mathbf{D}^{k} \mathbf{A}, \quad i=1,2, \ldots, N
$$

or, equivalently,

$$
Y^{(k)}=\left[\begin{array}{c}
y^{(k)}\left(x_{1}\right) \\
y^{(k)}\left(x_{2}\right) \\
\vdots \\
y^{(k)}\left(x_{N}\right)
\end{array}\right]=\mathbf{F D}^{k} \mathbf{A}
$$

Consequently, substituting (3.5) in equation (3.4), yields the fundamental matrix equation for problem (3.1),

$$
\sum_{k=1}^{n}\left(\mathbf{Q}_{k} \mathbf{F} \mathbf{D}^{k}\right) \mathbf{A}=\mathbf{G}, \quad n \leq N
$$

which corresponds to a system of $(N)$ algebraic equations for the unknown Fibonacci coefficients $a_{r}, r=1,2, \ldots, N$. In other words, when we denote the expression in the sum by $\mathbf{W}=\left[w_{r, s}\right]$ for $r=1,2, \ldots, N$ and $s=1,2, \ldots, N$, we get

$$
\mathbf{W A}=\mathbf{G} .
$$

Thus, the augmented matrix of equation (3.7) becomes

$[\mathbf{W}: \mathbf{G}]$. 
When the initial or boundary conditions are considered to be

$$
y^{(l)}\left(c_{j}\right)=\lambda_{j}, \quad j=1,2, \ldots, n-1, a \leq c_{j} \leq b
$$

it is seen from the relation (2.16), that

$$
\mathbf{U}_{j}=\left[y^{(l)}\left(c_{j}\right)\right]=\left[\lambda_{j}\right]
$$

where

$$
\begin{aligned}
\mathbf{U}_{j} & =\left[u_{\rho, \sigma}\right]=\mathbf{F}_{c_{j}} \mathbf{D}^{l} \mathbf{A} \text { and } \\
\mathbf{F}_{c_{j}} & =\left[\begin{array}{llll}
F_{1}\left(c_{j}\right) & F_{2}\left(c_{j}\right) & \cdots & F_{N}\left(c_{j}\right)
\end{array}\right] .
\end{aligned}
$$

Therefore, the augmented matrix of the specified conditions is

$$
\left[\mathbf{U}_{j}: \lambda_{j}\right]=\left[\begin{array}{llll}
u_{j_{1}} & u_{j_{2}} & \cdots & u_{j_{N}}: \lambda_{j}
\end{array}\right]
$$

Consequently, (3.8) together with (3.12) can be written in the new augmented matrix form

$$
\left[\mathbf{W}^{*}: \mathbf{G}^{*}\right]
$$

This form can also be achieved by replacing some rows of the matrix (3.8) by the rows of (3.12) or adding those rows to the matrix (3.8), provided that $\operatorname{det}\left(\mathbf{W}^{*}\right) \neq 0$. Finally, the vector $\mathbf{A}$ (thereby vector of the coefficients $a_{r}$ ) is determined by applying some numerical methods designed especially to solve the system of linear equations. On the other hand, when the singular case $\operatorname{det}\left(\mathbf{W}^{*}\right)=0$ appears, the least square methods are inevitably available to reach the best possible approximation. Therefore, the approximated solution can be obtained. This would be the Fibonacci series expansion of the solution to the problem (3.1) with specified conditions.

\section{Numerical results}

In this part, three illustrative examples have been shown in order to clarify the findings of the previous section. Then, the solutions $y^{a}(x)$ of the proposed method are compared with the exact solutions $y^{e}(x)$ in the tables and in the corresponding figures. It is noted here that the number of collocation points in the examples is indicated by the capital letter $N$.

Example 1 Consider the linear nonhomogeneous IVP [36],

$$
y^{\prime \prime}(x)+x y^{\prime}(x)-2 y(x)=x \cos x-3 \sin x
$$

with the initial conditions

$$
y(0)=0 \quad \text { and } \quad y^{\prime}(0)=1
$$

for which the exact solution is $y^{e}(x)=\sin x$.

Table 1 shows the absolute errors of the proposed method for $N=7$ and $N=9$. The approximation for $N=6$ and the exact solution are also plotted in Figure 1. 
Table 1 Absolute errors of Example 1 at different points

\begin{tabular}{lll}
\hline $\boldsymbol{x}$ & $\boldsymbol{N}=\mathbf{7}$ & $\boldsymbol{N}=\mathbf{9}$ \\
\hline-1 & $0.361 \mathrm{E}-04$ & $0.231 \mathrm{E}-06$ \\
-0.8 & $0.123 \mathrm{E}-05$ & $0.229 \mathrm{E}-07$ \\
-0.6 & $0.232 \mathrm{E}-05$ & $0.25 \mathrm{E}-08$ \\
-0.4 & $0.227 \mathrm{E}-05$ & $0.43 \mathrm{E}-08$ \\
-0.2 & $0.462 \mathrm{E}-06$ & $0.9 \mathrm{E}-09$ \\
0 & $0.305 \mathrm{E}-11$ & $0.762 \mathrm{E}-09$ \\
0.2 & $0.462 \mathrm{E}-06$ & $0.25 \mathrm{E}-08$ \\
0.4 & $0.227 \mathrm{E}-05$ & $0.58 \mathrm{E}-08$ \\
0.6 & $0.232 \mathrm{E}-05$ & $0.41 \mathrm{E}-08$ \\
0.8 & $0.123 \mathrm{E}-05$ & $0.245 \mathrm{E}-07$ \\
1 & $0.361 \mathrm{E}-04$ & $0.23 \mathrm{E}-06$ \\
\hline
\end{tabular}

\section{Figure 1 The proposed method solution for} $N=6$ and the exact solutions of Example 1 .

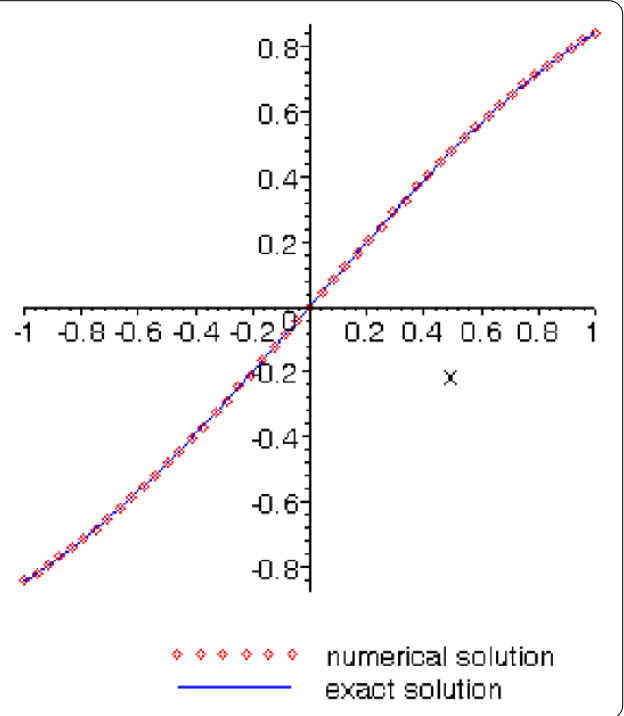

Table $2 L_{\infty}$ Errors of Example 2 for different $N$ values

\begin{tabular}{rlll}
\hline $\boldsymbol{N}$ & Present method & $\begin{array}{l}\text { Haar wavelet based } \\
\text { algorithm [37] }\end{array}$ & $\begin{array}{l}\text { B-spline wavelet } \\
\text { algorithm [38] }\end{array}$ \\
\cline { 2 - 4 } & $\boldsymbol{L}_{\infty}$ & $\boldsymbol{L}_{\infty}$ & $\boldsymbol{L}_{\infty}$ \\
\hline 9 & $9.715 \mathrm{E}-05$ & - & - \\
16 & $5.728 \mathrm{E}-07$ & $2.9051 \mathrm{E}-04$ & - \\
32 & $3.454 \mathrm{E}-08$ & $7.4812 \mathrm{E}-05$ & - \\
64 & $1.010 \mathrm{E}-08$ & $1.8956 \mathrm{E}-05$ & $2.5 \mathrm{E}-05$ \\
\hline
\end{tabular}

Example 2 Consider now a linear homogeneous BVP

$$
-y^{\prime \prime}=\left(2-4 x^{2}\right) y
$$

with Neumann boundary conditions

$$
y^{\prime}(0)=0 \text { and } y^{\prime}(1)=-2 / e \text {, }
$$

which is known to have the exact solution $y^{e}(x)=e^{-x^{2}}$.

Table 2 shows the comparison of the $L_{\infty}$ errors of the proposed approach, B-spline wavelet based algorithm [38] and Haar wavelet based algorithm [37]. The graphical repre- 


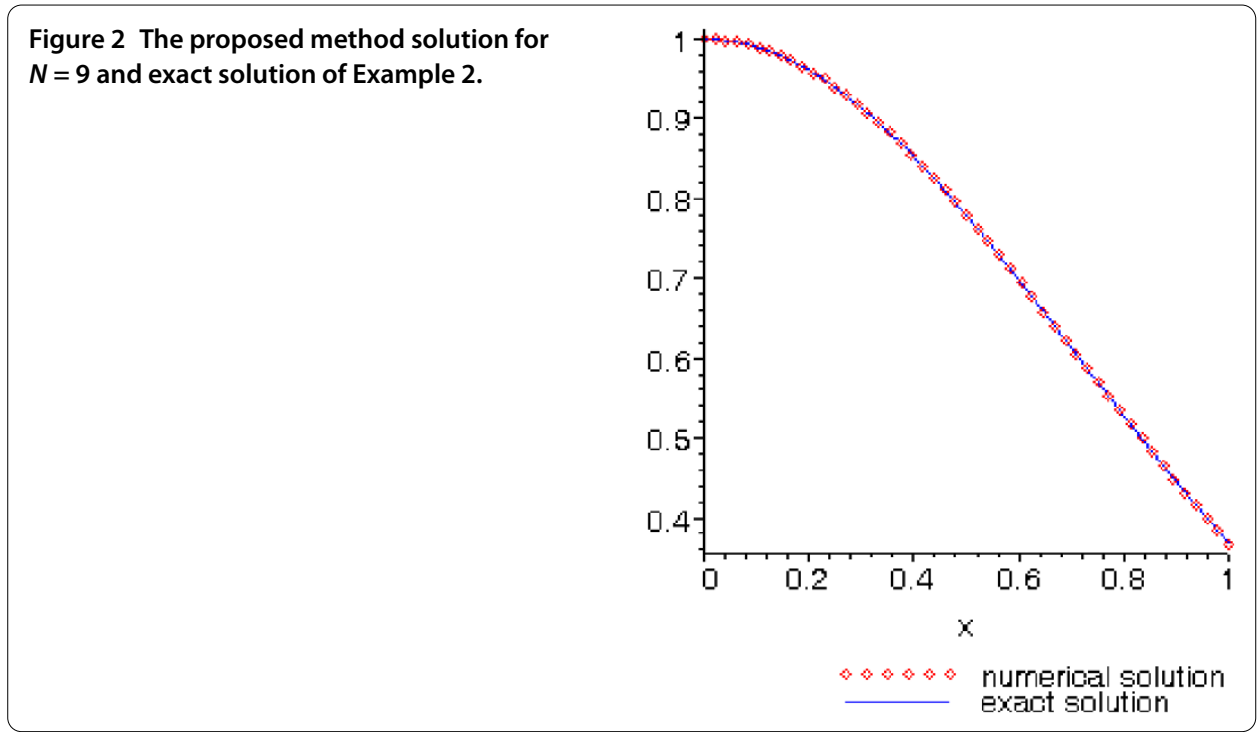

Table $3 L_{\infty}$ Errors of Example 3

\begin{tabular}{rll}
\hline $\boldsymbol{N}$ & Present method & $\begin{array}{l}\text { B-spline wavelet } \\
\text { algorithm [38] }\end{array}$ \\
\cline { 2 - 3 } & $\boldsymbol{L}_{\infty}$ & $\boldsymbol{L}_{\infty}$ \\
\hline 8 & $2.58 \mathrm{E}-06$ & - \\
16 & $8.94 \mathrm{E}-09$ & - \\
32 & $4.04 \mathrm{E}-09$ & $9.4 \mathrm{E}-05$ \\
64 & $2.09 \mathrm{E}-09$ & $2.0 \mathrm{E}-05$ \\
\hline
\end{tabular}

sentation of the approximation $y^{a}$ with the exact solution $y^{e}$ is also presented in Figure 2 . It is very promising to note that although $L_{\infty}$ errors of the solutions to the problem (4.2) for $N=64$ in $[37,38]$ are given $2.5 \mathrm{E}-05$ and 5.9E-06, respectively, almost the same level of error has been achieved by the Fibonacci approach as $9.22035 \mathrm{E}-05$ only for $N=9$.

Example 3 Consider a linear nonhomogeneous BVP

$$
y^{\prime \prime}(x)+4 y(x)=2, \quad x \in[0,1]
$$

with boundary conditions

$$
y^{\prime}(0)=0, \quad y^{\prime}(1)=\sin (2),
$$

whose exact solution is $y^{e}(x)=\sin ^{2}(x)$. A comparison of $L_{\infty}$ errors of our approach and B-spline wavelet algorithm [38] has been shown in Table 3 for different values of $N$. The exact and the approximate solutions of Example 3 for $N=9$ are plotted in Figure 3.

\section{Conclusion}

In this study, our aim is to propose a novel matrix method, based on the Fibonacci polynomials. Therefore, the operational matrices of derivative $\mathbf{D}$ and Fibonacci coefficient matrix $\mathbf{F}$ are introduced in the main body of the study. The matrix $\mathbf{D}$ is computationally very attractive, since it has few nonzeros above the main diagonal. Additionally, unlike the 


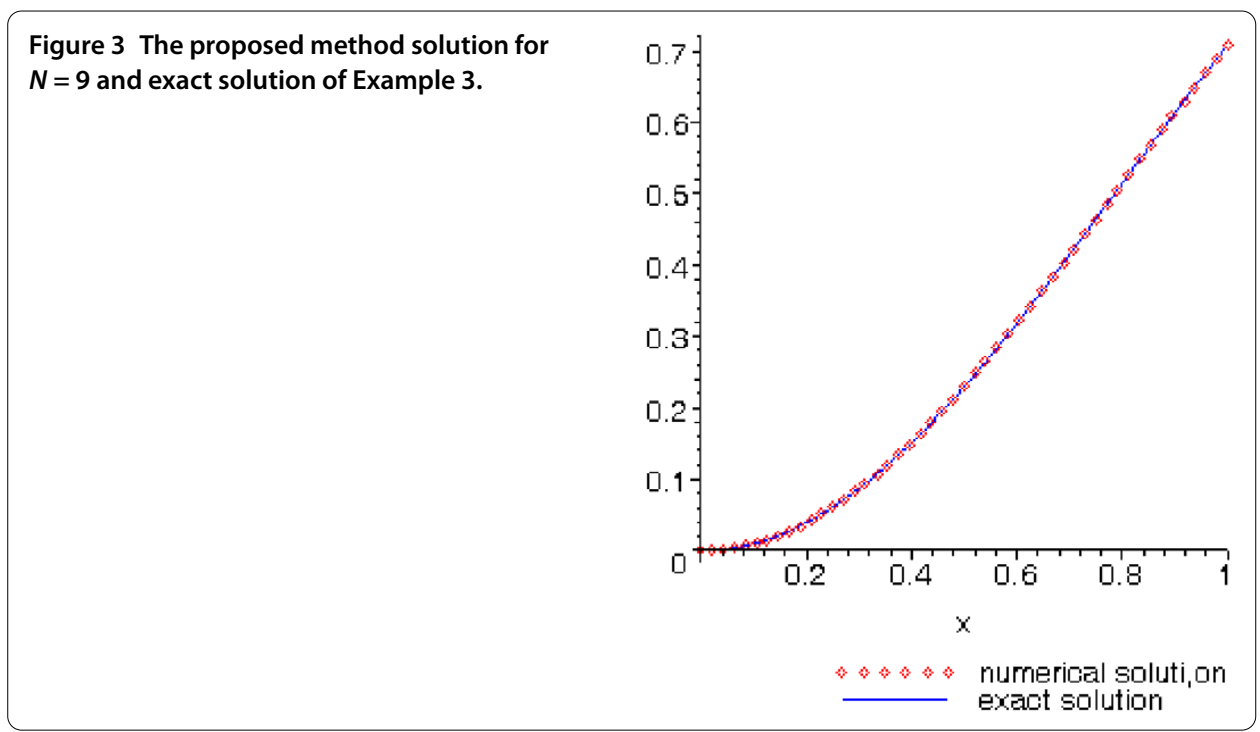

Chebyshev polynomial method, the Fibonacci approach does not require interval translation of the problem to an appropriate domain. Then, the reliability and efficiency of the method are verified by some illustrative examples of the boundary value problems. When the results are compared with some existing methods, the proposed method demonstrates its power in accuracy.

\section{Competing interests}

The authors declare that they have no competing interests.

Authors' contributions

The authors have equal contributions to each part of this article. All the authors read and approved the final manuscript.

\section{Author details}

'Department of Mathematics, Faculty of Science, Selcuk University, Konya, Turkey. ${ }^{2}$ Yayladagı Vocational School, Mustafa Kemal University, Hatay, Turkey.

\section{Acknowledgements}

The authors would like to thank the editor and referees for their valuable comments and remarks, which led to a great improvement of the article. Also, authors would like to thank the Selcuk University and TUBITAK for their support. Authors reveal here that this study was partially presented orally at the International Congress in Honour of Professor Hari M Srivastava, August 23-26, 2012, Bursa, Turkey.

Received: 10 May 2013 Accepted: 6 August 2013 Published: 27 August 2013

\section{References}

1. Lebedev, NN: Special Functions and Their Applications. Prentice Hall, London (1965) (Revised Eng. ed.: translated and edited by Silverman, RA (1972))

2. Yudell, LL: The Special Functions and Their Approximations, vol. 2. Mathematics in Science and Engineering (Bellman, R (ed.)), vol. 53. Academic Press, New York (1969)

3. Wang, ZX, Guo, DR: Special Functions. World Scientific, Singapore (1989)

4. Sahin, A, Dag, I, Saka, B: A B-spline algorithm for the numerical solution of Fisher's equation. Kybernetes 37(2), 326-342 (2008)

5. Doha, EH, Bhrawy, AH, Saker, MA: On the derivatives of Bernstein polynomials: an application for the solution of high even-order differential equations. Bound. Value Probl. 2011, 829543 (2011)

6. Celik, I, Gokmen, G: Approximate solution of periodic Sturm-Liouville problems with Chebyshev collocation method. Appl. Math. Comput. 170(1), 285-295 (2005)

7. Sezer, M, Karamete, A, Gulsu, M: Taylor polynomial solutions of systems of linear differential equations with variable coefficients. Int. J. Comput. Math. 82(6), 755-764 (2005)

8. Kadalbajoo, MK, Aggarwal, VK: Numerical solution of singular boundary value problems via Chebyshev polynomial and B-spline. Appl. Math. Comput. 160, 851-863 (2005)

9. Lamnii, A, Mraoui, H, Sbibih, D, Tijini, A, Zidna, A: Spline collocation method for solving linear sixth-order boundary value problems. Int. J. Comput. Math. 85(11), 1673-1684 (2008)

10. Lanczos, C: Trigonometric interpolation of empirical and analytic functions. J. Math. Phys. 17, 123-199 (1938) 
11. Fornberg, B: A Practical Guide to Pseudospectral Methods. Cambridge University Press, New York (1996)

12. Boyd, JP: Chebyshev and Fourier Spectral Methods, 2nd edn. Dover, New York (2000)

13. Canuto, C, Hussaini, MY, Quarteroni, A, Zang, TA: Spectral Methods: Fundamentals in Single Domains. Springer, Berlin (2006)

14. Agarwal, RP, O'Regan, D: Ordinary and Partial Differential Equations with Special Functions Fourier Series and Boundary Value Problems. Springer, New York (2009)

15. Sezer, M, Kaynak, M: Chebyshev polynomial solutions of linear differential equations. Int. J. Math. Educ. Sci. Technol. 27(4), 607-618 (1996)

16. Sezer, M, Doğan, S: Chebyshev series solutions of Fredholm integral equations. Int. J. Math. Educ. Sci. Technol. 27(5), 649-657 (1996)

17. Akyüz, A, Sezer, M: A Chebyshev collocation method for the solution of linear integro-differential equations. Int. J. Comput. Math. 72, 491-507 (1999)

18. Sezer, M, Kesan, C: Polynomial solutions of certain differential equations. Int. J. Comput. Math. 76(1), 93-104 (2000)

19. Mason, JC, Handscomb, DC: Chebyshev Polynomials. CRC Press, Boca Raton (2003)

20. Akyüz-Daşcıoğlu, A: Chebyshev polynomial solutions of systems of linear integral equations. Appl. Math. Comput. $151,221-232(2004)$

21. Celik, I: Solution of magnetohydrodynamic flow in a rectangular duct by Chebyshev collocation method. Int. J. Numer. Methods Fluids 66(10), 1325-1340 (2011)

22. Gurefe, N, Kocer, EG, Gurefe, Y: Chebyshev-Tau method for the linear Klein-Gordon equation. Int. J. Phys. Sci. 7(43), 5723-5728 (2012)

23. Bhrawy, AH, A-Shomrani, MM: A shifted Jacobi-Gauss-Lobatto collocation method for solving nonlinear fractional Langevin equation involving two fractional orders in different intervals. Bound. Value Probl. 2012, 62 (2012)

24. Li, H, Ma, H: Shifted Chebyshev collocation domain truncation for solving problems on an infinite interval. J. Sci. Comput. 18(2), 191-213 (2003)

25. Parand, K, Dehghan, M, Pirkhedri, A: Sinc-collocation method for solving the Blasius equation. Phys. Lett. A 373 4060-4065 (2009)

26. Yalcinbas, S, Aynigul, M, Sezer, M: A collocation method using Hermite polynomials for approximate solution of pantograph equations. J. Franklin Inst. 348, 1128-1139 (2011)

27. Kaya, B, Kurnaz, A, Koc, AB: Exponential Chebyshev Polynomials. Paper presented at the 3rd Conferences of the National Ereğli Vocational High School, University of Selcuk, Ereğli, 28-29 April (2011) (in Turkısh)

28. Boyd, JP: Rational Chebyshev spectral methods for unbounded solutions on an infinite interval using polynomial-growth special basis functions. Comput. Math. Appl. 41, 1293-1315 (2001)

29. Guo, B, Shen, J, Wang, Z: Chebyshev rational spectral and pseudospectral methods on a semi-infinite interval. Int. J. Numer. Methods Eng. 53, 65-84 (2002)

30. Sezer, M, Gülsu, M, Tanay, B: Rational Chebyshev collocation method for solving higher-order linear ordinary differential equations. Numer. Methods Partial Differ. Equ. 27(5), 1130-1142 (2011)

31. Gulsu, M, Gurbuz, B, Ozturk, Y, Sezer, M: Laguerre polynomial approach for solving linear delay difference equations. Appl. Math. Comput. 217, 6765-6776 (2011)

32. Byrd, PF: Expansion of analytic functions in polynomials associated with Fibonacci numbers. Fibonacci Q. 1(1), 12-29 (1963)

33. Horadam, AF, Mahon, BJM: Pell and Pell-Lucas polynomials. Fibonacci Q. 23(1), 7-20 (1985)

34. Falcon, S, Plaza, A: The k-Fibonacci sequence and the Pascal 2-triangle. Chaos Solitons Fractals 33(1), 38-49 (2007)

35. Falcon, S, Plaza, A: On k-Fibonacci sequences and polynomials and their derivatives. Chaos Solitons Fractals 39 1005-1019 (2009)

36. Sezer, M, Gulsu, M: Solving high-order linear differential equations by a Legendre matrix method based on hybrid Legendre and Taylor polynomials. Numer. Methods Partial Differ. Equ. 26(3), 647-661 (2010)

37. Siraj-ul-Islam, Aziz, I, Sarler, B: The numerical solution of second-order boundary-value problems by collocation method with the Haar wavelets. Math. Comput. Model. 52, 1577-1590 (2010)

38. Lakestani, M, Dehghan, M: The solution of a second-order nonlinear differential equation with Neumann boundary conditions using semi-orthogonal B-spline wavelets. Int. J. Comput. Math. 83(8-9), 685-694 (2006)

doi:10.1186/1687-1847-2013-262

Cite this article as: Koç et al.: A new Fibonacci type collocation procedure for boundary value problems. Advances in Difference Equations 2013 2013:262.

\section{Submit your manuscript to a SpringerOpen ${ }^{\circ}$ journal and benefit from:}

- Convenient online submission

- Rigorous peer review

- Immediate publication on acceptance

- Open access: articles freely available online

- High visibility within the field

- Retaining the copyright to your article

Submit your next manuscript at $>$ springeropen.com 